Journal of Science $\quad$ Gazi University

\title{
Generalization for the Average Value of the Difference Between the Energies of Two Graphs
}

Ilkay ALTINDAG

Department of Banking, Faculty of Applied Sciences, Necmettin Erbakan University, 42090, Konya, Turkey

\section{Highlights}

- We determine the average values of the difference between $A$-energies of two graphs.

- Our results yield criterions for deciding when two graphs are almost $A$-equienergetic.

- Our results also generalize some previous results.

\section{Article Info}

Received: $26 / 02 / 2019$

Accepted: 01/10/2019

\section{Keywords}

(A-) Energy of Graph

Almost (A-)

Equienergetic Graphs

\begin{abstract}
Let $\hat{G}_{n, m}$ denote the set of all simple graphs with $n$ vertices and $m$ edges. In this paper, for a given type of graph Hermite matrix $A$, we determine the average values of the difference between $A$ energies of two graphs randomly chosen from $\hat{G}_{n, m}$. These results yield criterions for deciding when two graphs are almost $A$-equienergetic. Our results generalize some previous results in the literature. Moreover, we give new results on Laplacian energy.
\end{abstract}

\section{INTRODUCTION}

Let $G$ be a simple graph with $n$ vertices labeled as $v_{1}, v_{2}, \ldots, v_{n}$ and $m$ edges. The degree of the vertex $v_{i}$, denoted by $d_{i}$, is equal to the number of vertices adjacent to $v_{i}$. Let $A(G)$ be the $(0,1)$-adjacency matrix of $G$ with eigenvalues $\lambda_{1}, \lambda_{2}, \ldots, \lambda_{n}$. The eigenvalues of $A(G)$ are said to be the eigenvalues of $G$ and to form its spectrum [1]. The energy of a graph $G$, denoted by $E(G)$, is defined as the sum of absolute values of its eigenvalues, i.e., [2]

$$
E=E(G)=\sum_{i=1}^{n}\left|\lambda_{i}\right|
$$

The concept of graph energy has important applications in molecular orbital theory of organic molecules $[3,4]$. It is used for estimation on the total $\pi$-electron energy of conjugated hydrocarbons [5]. For more information on $E(G)$, see [6-9].

Two non-isomorphic graphs are said to be cospectral if they have same eigenvalues [1]. Any two graphs $G_{1}$ and $G_{2}$ having same number of vertices are said to be equienergetic if $E\left(G_{1}\right)=E\left(G_{2}\right)$ [10-12]. Cospectral graphs are clearly equienergetic. Recently, several authors determined the constructions of noncospectral equienergetic graphs [13-16]. Furthermore, there exists graphs whose energies are different but remarkably close. These type of graphs are said to be almost equienergetic graphs $[10,15]$. At first, 
Graovac et al. [10] gave some theoretical results for almost equienergeticity using the average value of the difference between the energies of two graphs. According to the numerical experience, the authors [15] took the criterion for almost equienergeticity of two graphs $G_{1}$ and $G_{2}$ such that $0<\left|E\left(G_{1}\right)-E\left(G_{2}\right)\right|<10^{-8}$ and they gave detailed study on almost equienergetic trees. In [17], Stanić and Gutman showed that the limit value $10^{-8}$ is arbitrary and unjustified and presented some arguments in favor of possibility that the difference $E\left(G_{1}\right)-E\left(G_{2}\right)$ can be much smaller. For more details on almost equienergetic graphs, see [18]. Let $D(G)=\operatorname{diag}\left(d_{1}, d_{2}, \ldots, d_{n}\right)$ be the diagonal matrix of the vertex degrees of the graph $G$. The Laplacian matrix of $G$ is defined as $L(G)=D(G)-A(G)$ with $\mu_{1} \geq \mu_{2} \geq \ldots \geq \mu_{n}=0$, where $A(G)$ is the adjacency matrix of $G$ [19]. In [20], Gutman and Zhou extended the concept of ordinary graph energy and defined the Laplacian energy of $G$ as

$$
L E=L E(G)=\sum_{i=1}^{n}\left|\mu_{i}-\frac{2 m}{n}\right| .
$$

For survey and more information on $L E(G)$, see [21-23].

In full analogy with graph energy, Laplacian equienergetic and almost Laplacian equienergetic graphs are defined and studied in [15]. For details on these graphs, see the recent papers [24-26].

Recall that [27] a square matrix $A=\left(a_{i, j}\right)$ such that $A^{*}=A$ is said to be Hermite matrix, where $A^{*}$ denotes the conjugate transpose of $A$. This is well known fact that the eigenvalues of Hermite matrix $A$ are real numbers and can be ordered according to decreasing size [27]. In [28], Liu and Liu generalized the concepts of graph energy and Laplacian energy to any Hermite matrix $A$ as follows: For an $n \times n$ Hermite matrix $A$ with eigenvalues $\gamma_{1} \geq \gamma_{2} \geq \ldots \geq \gamma_{n}$, the energy of $A$ or $A$-energy is defined as [28]

$$
H E=H E(A)=\sum_{i=1}^{n}\left|\gamma_{i}-\frac{\operatorname{tr}(A)}{n}\right|
$$

where $\operatorname{tr}(A)$ is the trace of the matrix $A$. Throughout this paper instead of $\operatorname{tr}(A)$, we shortly use the notion $T$.

Let $I_{n}$ be the $n \times n$ identity matrix. If $A$ is a Hermite matrix, then $A-\frac{T}{n} I_{n}$ is also a Hermite matrix [28].

Let $\xi_{1} \geq \xi_{2} \geq \ldots \geq \xi_{n}$ be the eigenvalues of $A-\frac{T}{n} I_{n}$. Obviously, $\xi_{i}+\frac{T}{n}=\gamma_{i}$, that is, $\xi_{i}=\gamma_{i}-\frac{T}{n}$ [28]. Then, Equation (3) turns into [28]

$$
H E=H E(A)=\sum_{i=1}^{n}\left|\xi_{i}\right|
$$

Specially for a graph $G$, if $A$ is the adjacency matrix (whose trace is 0 ) of $G$, then $H E(A)=E(G)$ [28]. If $A$ is the Laplacian matrix (whose trace is equal to $2 m$ ) of $G$, then $H E(A)=L E(G)$ [28].

For a given type of graph Hermite matrix $A$, two graphs are said to be $A$-equienergetic if they are not $A$ cospectral, yet have equal $A$-energies [26]. Moreover, if the difference between $A$-energies of two graphs is very small, we call these graphs as almost $A$-equienergetic. In this paper, we determine the average values of this difference in order to decide when the difference is very small. Our results are natural generalization of the results obtained in [10,29] and give new results on Laplacian energy [20]. 


\section{PRELIMINARIES}

In this section, we give some preliminaries which will be used in the subsequent section.

Lemma 2.1. [28] Let $A=\left(a_{i j}\right)$ be an $n \times n$ Hermite matrix and let $S=\sum_{i, j=1}^{n}\left|a_{i j}\right|^{2}$, where $\left|a_{i j}\right|$ is the modulo of $a_{i j}$. Then

$\sum_{i=1}^{n} \gamma_{i}=T, \sum_{i=1}^{n} \gamma_{i}^{2}=S, \sum_{i=1}^{n} \xi_{i}=0$ and $\sum_{i=1}^{n} \xi_{i}^{2}=S-\frac{T^{2}}{n}$.

For the case of adjacency spectrum and Laplacian spectrum in Lemma 2.1, the following corollary was given in [28].

Corollary 2.2. $[4,20,28]$

$\sum_{i=1}^{n} \lambda_{i}=0 ; \sum_{i=1}^{n} \mu_{i}=2 m ; \sum_{i=1}^{n}\left(\mu_{i}-\frac{2 m}{n}\right)=0$

$\sum_{i=1}^{n} \lambda_{i}^{2}=S=2 m ; \sum_{i=1}^{n} \mu_{i}^{2}=S=2 m+\sum_{i=1}^{n} d_{i}^{2}$;

and

$\sum_{i=1}^{n}\left(\mu_{i}-\frac{2 m}{n}\right)^{2}=S-\frac{T^{2}}{n}=2\left[m+\frac{1}{2} \sum_{i=1}^{n}\left(d_{i}-\frac{2 m}{n}\right)^{2}\right] \stackrel{\text { def }}{=} 2 M$.

Using Lemma 2.1, one can easily arrive at the following result.

Lemma 2.3. Let $A=\left(a_{i j}\right)$ be an $n \times n$ Hermite matrix with eigenvalues $\gamma_{1} \geq \gamma_{2} \geq \ldots \geq \gamma_{n}$. Let $P=\sum_{i=1}^{n} \gamma_{i}^{3}$ and $S$ and $T$ be given as in Lemma 2.1. Then

$\sum_{i=1}^{n} \xi_{i}^{3}=P-\frac{3 S T}{N}+\frac{2 T^{3}}{n^{2}}$.

Let $t$ denote the number of triangles of a graph $G$. For the case of adjacency and Laplacian matrices of $G$ in Lemma 2.3, we have the following observation:

\section{Corollary 2.4.}

$\sum_{i=1}^{n} \gamma_{i}^{3}=P=6 t$ and $\sum_{i=1}^{n} \mu_{i}^{3}=P=\sum_{i=1}^{n} d_{i}^{3}+3 \sum_{i=1}^{n} d_{i}^{2}-6 t$, see [30].

Combining this with Corollary 2.2,

$$
\begin{aligned}
\sum_{i=1}^{n}\left(\mu_{i}-\frac{2 m}{n}\right)^{3} & =P-\frac{3 S T}{N}+\frac{2 T^{3}}{n^{2}} \\
& =\sum_{i=1}^{n} d_{i}^{3}+\left(3-\frac{6 m}{n}\right) \sum_{i=1}^{n} d_{i}^{2}+\frac{16 m^{3}}{n^{2}}-\frac{12 m^{2}}{n}-6 t \stackrel{\text { def }}{=} Q .
\end{aligned}
$$

Let $\hat{G}_{n, m}$ be the set of all simple graphs with $n$ vertices and $m$ edges and let $G_{1}$ and $G_{2} \in \hat{G}_{n, m}$. Let $A_{1}$ and $A_{2}$ be the same kind of Hermite matrices of $G_{1}$ and $G_{2}$, respectively. The average value of the difference $\left|H E\left(A_{1}\right)-H E\left(A_{2}\right)\right|$ will be denoted by $\tilde{E}\left(\left|H E\left(A_{1}\right)-H E\left(A_{2}\right)\right|\right)$. In here, the averaging is taken over all pairs of the elements in $\hat{G}_{n, m}$. Instead of $\tilde{E}\left(\left|H E\left(A_{1}\right)-H E\left(A_{2}\right)\right|\right)$ we handle the following quantity

$\sqrt{\tilde{E}\left(\left(H E\left(A_{1}\right)-H E\left(A_{2}\right)\right)^{2}\right)}$

whose statistical analysis is simpler. Indeed,

$\tilde{E}\left(H E\left(A_{1}\right)\right)=\tilde{E}\left(H E\left(A_{2}\right)\right)=\tilde{E}(H E)$

and 


$$
\tilde{E}\left(\left(H E\left(A_{1}\right)\right)^{2}\right)=\tilde{E}\left(\left(H E\left(A_{2}\right)\right)^{2}\right)=\tilde{E}\left(H E^{2}\right) .
$$

Since $\operatorname{HE}\left(A_{1}\right)$ and $H E\left(A_{2}\right)$ are considered as independent random variables,

$$
\tilde{E}\left(H E\left(A_{1}\right) \cdot H E\left(A_{2}\right)\right)=\tilde{E}\left(H E\left(A_{1}\right)\right) \cdot \tilde{E}\left(H E\left(A_{2}\right)\right)=(\tilde{E}(H E))^{2} \text {. }
$$

Therefore

$$
\tilde{E}\left(\left(H E\left(A_{1}\right)-H E\left(A_{2}\right)\right)^{2}\right)=2\left[\tilde{E}\left(H E^{2}\right)-(\tilde{E}(H E))^{2}\right]
$$

where $\tilde{E}(H E)$ and $\tilde{E}\left(H E^{2}\right)$ denote the average values of the energy pertaining to any Hermite matrix of the graph and its square, respectively, averaged over all elements in $\hat{G}_{n, m}$.

\section{MAIN RESULTS}

Considering the similar techniques in [10], we now present general results which cover the energies of all Hermite matrices of graphs. For a given type of Hermite matrix $A$ of a simple graph $G$ with $n$ vertices and $m$ edges, recall that $\xi_{1} \geq \xi_{2} \geq \ldots \geq \xi_{n}$ denote the eigenvalues of $A-\frac{T}{n} I_{n}$, where $I_{n}$ is $n \times n$ identity matrix. Using Eq. (4), we first give an approximate relation between $\tilde{E}(H E)$ and $\tilde{E}\left(H E^{2}\right)$, averaged over all elements in $\hat{G}_{n, m}$. In order to do this, we assume that $\xi_{1}, \xi_{2}, \ldots, \xi_{n}$ are replaced by the random variables $x_{1}, x_{2}, \ldots, x_{n}$ with an arbitrary probability distrubition which is the same for each $x_{i}$.

Theorem 3.1. Let $\tilde{E}(H E)$ and $\tilde{E}\left(H E^{2}\right)$ be the average values of the energy pertaining to any Hermite matrix $A$ of the graph and its square, respectively. Let $S$ and $T$ be given as in Lemma 2.1. Then

$$
\tilde{E}\left(H E^{2}\right) \approx S-\frac{T^{2}}{n}+\frac{n-1}{n}(\tilde{E}(H E))^{2} \text {. }
$$

Proof. We start with taking the average value of the square of the energy pertaining to Hermite matrix $A$

$$
\begin{aligned}
\tilde{E}\left(H E^{2}\right) & =\tilde{E}\left(\sum_{i=1}^{n} \xi_{i}^{2}+2 \sum_{i<j}\left|\xi_{i}\right|\left|\xi_{j}\right|\right) \\
& =S-\frac{T^{2}}{n}+2\left(\sum_{i<j} \tilde{E}\left(\left|\xi_{i}\right|\right) \tilde{E}\left(\left|\xi_{j}\right|\right)\right),
\end{aligned}
$$

by Lemma 2.1 , for all graphs in $\hat{G}_{n, m}$,

$$
\sum_{i=1}^{n} \xi_{i}^{2}=S-\frac{T^{2}}{n}
$$

By replacing $\xi_{i}$ and $\xi_{j}$ with $x_{i}$ and $x_{j}$ and bearing in mind these two variables are statistically independent such that $\tilde{E}\left(\left|x_{i}\right|\right)=\tilde{E}\left(\left|x_{j}\right|\right)=\tilde{E}(|x|)$, we obtain

$$
\tilde{E}\left(H E^{2}\right) \approx S-\frac{T^{2}}{n}+\frac{n-1}{n}(n \tilde{E}(|x|))^{2} .
$$

Clearly,

$$
\tilde{E}(H E)=\tilde{E}\left(\sum_{i=1}^{n}\left|\xi_{i}\right|\right)=\sum_{i=1}^{n} \tilde{E}\left(\left|\xi_{i}\right|\right) \approx \sum_{i=1}^{n} \tilde{E}\left(\left|x_{i}\right|\right)=n \tilde{E}(|x|) \text {. }
$$

Then, by (9) and (10), we have

$$
\tilde{E}\left(H E^{2}\right) \approx S-\frac{T^{2}}{n}+\frac{n-1}{n}(\tilde{E}(H E))^{2} \text {. }
$$

Hence, we complete the proof of theorem.

By Corollary 2.2, for the adjacency matrices of the graphs in $\hat{G}_{n, m}, S=2 m$ and $T=0$. Therefore, from Theorem 3.1, we have the following result. 
Corollary 3.2. [10] Let $\tilde{E}(E)$ and $\tilde{E}\left(E^{2}\right)$ be the average values of the ordinary graph energy and its square, respectively. Then

$\tilde{E}\left(E^{2}\right) \approx 2 m+\frac{n-1}{n}(\tilde{E}(E))^{2}$.

By Corollary 2.2, for the Laplacian matrices of the graphs in $\hat{G}_{n, m}, S=2 m+\sum_{i=1}^{n} d_{i}^{2}, T=2 m$ and $S-\frac{T^{2}}{n}=2\left[m+\frac{1}{2} \sum_{i=1}^{n}\left(d_{i}-\frac{2 m}{n}\right)^{2}\right]=2 M$. Then, from Theorem 3.1, we have the following observation:

Corollary 3.3. Let $\tilde{E}(L E)$ and $\tilde{E}\left(L E^{2}\right)$ be the average values of the Laplacian energy and its square, respectively. Then

$$
\tilde{E}\left(L E^{2}\right) \approx 2 M+\frac{n-1}{n}(\tilde{E}(L E))^{2} .
$$

Note that we get relation (7) without specifying the actual distribution of the random variables $x_{1}, x_{2}, \ldots, x_{n}$ . If we want to obtain the average difference (5) from (6) and (7), then we need to know this distrubution, at least approximately. For this, we utilize the probability function $\Gamma(x)$ of the random variables $x_{1}, x_{2}, \ldots, x_{n}$. Using the function $\Gamma(x)$, we present a statistical model for the actual distrubution of the eigenvalues $\xi_{1}, \xi_{2}, \ldots, \xi_{n}$ associated with any type of Hermite matrix of the graphs in $\hat{G}_{n, m}$. Note that $\Gamma(x)$ must satisfy the following conditions [10]:

$\Gamma(x) \geq 0$, for all $x \in(-\infty,+\infty)$

and

$\int_{-\infty}^{+\infty} \Gamma(x) d x=1$

By Lemma 2.1, for all graphs in $\hat{G}_{n, m}, \sum_{i=1}^{n} \xi_{i}=0$. Then, we require that

$\int_{-\infty}^{+\infty} x \Gamma(x) d x=0$

By (4) and (10)

$\tilde{E}(H E)=n \int_{-\infty}^{+\infty}|x| \Gamma(x) d x$.

Theorem 3.4. Let $G_{1}$ and $G_{2}$ be two graphs randomly chosen from the set $\hat{G}_{n, m}$ and $A_{1}$ and $A_{2}$ be the same kind of Hermite matrices of $G_{1}$ and $G_{2}$, respectively. Further let $S$ and $T$ be given as in Lemma 2.1. Then

$$
\tilde{E}\left(\left|H E\left(A_{1}\right)-H E\left(A_{2}\right)\right|\right) \approx \sqrt{\frac{1}{2}\left(S-\frac{T^{2}}{n}\right)} .
$$

Proof. In this proof, we utilize the following uniform-distrubition for $\Gamma(x)$ [10]

$$
\Gamma\left(x_{i}\right)=\Gamma(x)=\left\{\begin{array}{cl}
1 /(2 \alpha) & \text { if }|x| \leq \alpha \\
0 & \text { if }|x|>\alpha
\end{array}\right.
$$

Note that $\Gamma(x)$ satisfies the conditions (13)-(15) and the parameter $\alpha$ in (18) will be determined with the condition

$$
\int_{-\infty}^{+\infty} x^{2} \Gamma(x) d x=\frac{S-\frac{T^{2}}{n}}{n}
$$


This gives just a reformulation of (8). In (19), the quantity $S-\frac{T^{2}}{n}$ must be considered as the average $S-\frac{T^{2}}{n}$ value of the graphs in $\hat{G}_{n, m}$. By (16), (18) and (19), we obtain that $\alpha=\sqrt{\frac{3\left(S-\frac{T^{2}}{n}\right)}{n}}$ and so $\tilde{E}(H E) \approx \frac{\sqrt{3}}{2} \sqrt{n\left(S-\frac{T^{2}}{n}\right)}$.

Then by (6), (7) and (20)

$\tilde{E}\left(\left|H E\left(A_{1}\right)-H E\left(A_{2}\right)\right|\right) \approx \sqrt{\frac{1}{2}\left(S-\frac{T^{2}}{n}\right)}$.

Hence the required result holds.

From Theorem 3.4, we particularly have the following corollaries.

Corollary 3.5. [10] By Corollary 2.2, for the adjacency matrices of the graphs in $\hat{G}_{n, m}, S=2 m$ and $T=0$ . Thus

$\tilde{E}\left(\left|E\left(G_{1}\right)-E\left(G_{2}\right)\right|\right) \approx \sqrt{m}$.

Corollary 3.6. By Corollary 2.2, for the Laplacian matrices of the graphs in $\hat{G}_{n, m}, S=2 m+\sum_{i=1}^{n} d_{i}^{2}, T=2 m$ and $S-\frac{T^{2}}{n}=2\left[m+\frac{1}{2} \sum_{i=1}^{n}\left(d_{i}-\frac{2 m}{n}\right)^{2}\right]=2 M$

Therefore

$\tilde{E}\left(\left|L E\left(G_{1}\right)-L E\left(G_{2}\right)\right|\right) \approx \sqrt{M}$

Theorem 3.7. Let $G_{1}$ and $G_{2}$ be two graphs randomly chosen from the set $\hat{G}_{n, m}$ and $A_{1}$ and $A_{2}$ be the same kind of Hermite matrices of $G_{1}$ and $G_{2}$, respectively. Further let $P, S$ and $T$ be given as in Lemmas 2.1 and 2.3. Then

$$
\tilde{E}\left(\left|H E\left(A_{1}\right)-H E\left(A_{2}\right)\right|\right) \approx \sqrt{\frac{1}{2}\left(S-\frac{T^{2}}{n}\right)+\frac{6 n\left(S-\frac{T^{2}}{n}\right)\left(P-\frac{3 S T}{n}+\frac{2 T^{3}}{n^{2}}\right)^{2}}{4 n\left(P-\frac{3 S T}{n}+\frac{2 T^{3}}{n^{2}}\right)^{2}+27\left(S-\frac{T^{2}}{n}\right)^{3}}}
$$

Proof. In the proof, we use the following model for $\Gamma(x)$ which is given in [10]

$\Gamma\left(x_{i}\right)=\Gamma(x)= \begin{cases}h_{\beta} & \text { if }-\beta \leq x \leq 0 \\ h_{\alpha} & \text { if } 0<x \leq \alpha \\ 0 & \text { otherwise }\end{cases}$

Note that the parameters $\alpha, \beta, h_{\alpha}$ and $h_{\beta}$ in (24) will be found from the conditions (14), (15), (19) and (25)

$\int_{-\infty}^{+\infty} x^{3} \Gamma(x) d x=\frac{P-\frac{3 S T}{n}+\frac{2 T^{3}}{n^{2}}}{n}$ 
which is a reformulation of the identity given in Lemma 2.3. In (25), the quantity $P-\frac{3 S T}{n}+\frac{2 T^{3}}{n^{2}}$ must be considered as the average $P-\frac{3 S T}{n}+\frac{2 T^{3}}{n^{2}}$ value of the graphs in $\hat{G}_{n, m}$. From (14) and (15), Graovac et al. obtained [10]

$h_{\alpha}=\frac{\beta}{\alpha(\alpha+\beta)} ; h_{\beta}=\frac{\alpha}{\beta(\alpha+\beta)}$.

By (19) and (25), we get

$\alpha \beta=\frac{3\left(S-\frac{T^{2}}{n}\right)}{n} ; \alpha-\beta=\frac{4\left(P-\frac{3 S T}{n}+\frac{2 T^{3}}{n^{2}}\right)}{3\left(S-\frac{T^{2}}{n}\right)}$

from which

$$
\begin{aligned}
& \alpha=\frac{2\left(P-\frac{3 S T}{n}+\frac{2 T^{3}}{n^{2}}\right)}{3\left(S-\frac{T^{2}}{n}\right)}+\sqrt{\frac{4\left(P-\frac{3 S T}{n}+\frac{2 T^{3}}{n^{2}}\right)^{2}}{9\left(S-\frac{T^{2}}{n}\right)^{2}}+\frac{3\left(S-\frac{T^{2}}{n}\right)}{n}} ; \\
& \beta=-\frac{2\left(P-\frac{3 S T}{n}+\frac{2 T^{3}}{n^{2}}\right)}{3\left(S-\frac{T^{2}}{n}\right)}+\sqrt{\frac{4\left(P-\frac{3 S T}{n}+\frac{2 T^{3}}{n^{2}}\right)^{2}}{9\left(S-\frac{T^{2}}{n}\right)^{2}}+\frac{3\left(S-\frac{T^{2}}{n}\right)}{n}} .
\end{aligned}
$$

By (16), (24) and (26)-(28), we also get

$$
\tilde{E}(H E)=\frac{3\left(S-\frac{T^{2}}{n}\right)}{2}\left(\frac{4\left(P-\frac{3 S T}{n}+\frac{2 T^{3}}{n^{2}}\right)^{2}}{9\left(S-\frac{T^{2}}{n}\right)^{2}}+\frac{3\left(S-\frac{T^{2}}{n}\right)}{n}\right)^{-1 / 2} \text {. }
$$

Taking into account this result with (6) and (7), we obtain the required result.

From Theorem 3.7, we particularly have the following results.

Corollary 3.8. [10,29] By Corollaries 2.2 and 2.4, for the adjacency matrices of the graphs in $\hat{G}_{n, m}, P=6 t, S=2 m$ and $T=0$. Thus

$\tilde{E}\left(\left|E\left(G_{1}\right)-E\left(G_{2}\right)\right|\right) \approx \sqrt{m+\frac{6 m n t^{2}}{3 m^{3}+2 n t^{2}}}$.

Corollary 3.9. By Corollaries 2.2 and 2.4 , for the Laplacian matrices of the graphs in $\hat{G}_{n, m}$,

$$
\begin{aligned}
& P=\sum_{i=1}^{n} d_{i}^{3}+3 \sum_{i=1}^{n} d_{i}^{2}-6 t ; S=2 m+\sum_{i=1}^{n} d_{i}^{2} ; T=2 m \\
& P-\frac{3 S T}{n}+\frac{2 T^{3}}{n^{2}}=\sum_{i=1}^{n} d_{i}^{3}+\left(3-\frac{6 m}{n}\right) \sum_{i=1}^{n} d_{i}^{2}+\frac{16 m^{3}}{n^{2}}-\frac{12 m^{2}}{n}-6 t=Q
\end{aligned}
$$

and

$$
S-\frac{T^{2}}{n}=2\left[m+\frac{1}{2} \sum_{i=1}^{n}\left(d_{i}-\frac{2 m}{n}\right)^{2}\right]=2 M .
$$


Therefore

$$
\tilde{E}\left(\left|L E\left(G_{1}\right)-L E\left(G_{2}\right)\right|\right) \approx \sqrt{M+\frac{3 M n Q^{2}}{54 M^{3}+n Q^{2}}} .
$$

Remark 3.10. We point out that (17) gives somewhat better estimation than (23) when $P-\frac{3 S T}{n}+\frac{2 T^{3}}{n^{2}} \neq 0$. Moreover, it is easy to see that (23) reduces to (17) when $P-\frac{3 S T}{n}+\frac{2 T^{3}}{n^{2}}=0$.

\section{CONCLUSIONS}

In this paper, we present an approximate relation between the average values of the energy pertaining to any Hermite matrix of the graph and its square, averaged over all elements in $\hat{G}_{n, m}$. Using this result, for a given type of graph Hermite matrix $A$, we determine the average values of the difference between $A$ energies of two graphs randomly chosen from $\hat{G}_{n, m}$. These results generalize the results obtained in [10, 29] on graph energy [2] and Randić energy [31] and give new results on Laplacian energy [20]. Note that various analogous properties between the ordinary graph energy and Laplacian energy have been recently discovered in [20, 32]. From the results given in (11), (12), (21), (22), (29) and (30), we conclude that these results are also similarities between the graph energy and Laplacian energy.

Finally, it is worth to mention that signless Laplacian energy, distance energy and etc. [6, 8, 33, 34] are also the special cases of the energy of Hermite matrix of a graph. Therefore all main results can be directly applied to these graph energies.

\section{CONFLICT OF INTEREST}

No conflict of interest was declared by the author.

\section{REFERENCES}

[1] Cvetković, D., Doob, M., Sachs, H., Spectra of Graphs-Theory and Application, Academic Press, New York, (1980).

[2] Gutman, I., "The energy of a graph", Ber. Math. Stat. Sekt. Forschungsz. Graz, 103: 1-22, (1978).

[3] Gutman, I., "Total $\pi$-electron energy of benzenoid hydrocarbons", Topics Curr. Chem., 162: 29-63, (1992).

[4] Gutman, I., "The energy of a graph: old and new results", In: Betten, A., Kohnert, A., Laue, R., Wassermann A. ed., Algebraic Combinatorics and Applications, Springer-Verlag, Berlin, 196-211, (2001).

[5] Gutman, I., "Topology and stability of conjugated hydrocarbons. The dependence of total $\pi$-electron energy on molecular topology", J. Serb. Chem. Soc. 70: 441-456, (2005).

[6] Gutman, I., "Comparative studies of graph energies", Bulletin de l'Acad'emie Serbe des Sciences et des Arts (Classe des Sciences Math'ematiques et Naturelles), 144: 1-17, (2012).

[7] Gutman, I., Li, X., Zhang, J., "Graph energy", In: Dehmer, M., Emmert-Streib F. ed., Analysis of Complex Networks From Biology to Linguistics, Wiley-VCH, Weinheim, 145-174, (2009).

[8] Li, X., Shi, Y., Gutman, I., Graph Energy, Springer, New York, (2012). 
[9] Ganie, H. A., Samee, U. T., Pirzada, S. “On graph energy, maximum degree and vertex cover number”, Le Matematiche, 74: 163-172, (2019).

[10] Graovac, A., Gutman, I., John, P.E., Vidović, D., Vlah, I., "On statistics of graph energy", Z. Naturforsch., 56a: 307-311, (2001).

[11] Balakrishnan, R., "The energy of a graph", Lin. Algebra Appl., 387: 287-295, (2004).

[12] Brankov, V., Stevanović, D., Gutman, I., "Equienergetic chemical trees", J. Serb. Chem. Soc., 69: 549-553, (2004).

[13] Ganie, H. A., Pirzada, L., Iványi, A., "Energy, Laplacian energy of double graphs and new families of equienergetic graphs", Acta Univ. Sapientiae Informatica, 6: 89-116, (2014).

[14] Indulal, G., Vijayakumar, A., "On a pair of equienergetic graphs", MATCH Commun. Math. Comput. Chem., 55: 83-90, (2006).

[15] Miljković, O., Furtula, B., Radenković, S., Gutman, I., "Equienergetic and almost-equienergetic trees", MATCH Commun. Math. Comput. Chem., 61: 451-461, (2009).

[16] Ramane, H.S., Walikar, H.B., "Construction of equienergetic graphs", MATCH Commun. Math. Comput. Chem., 57: 203-210, (2007).

[17] Stanić, M.P., Gutman, I., "On almost-equienergetic graphs", MATCH Commun. Math. Comput. Chem., 70: 681-688, (2013).

[18] Stanić, M.P., Gutman, I., "Towards a definition of almost-equienergetic graphs", J. Math. Chem., 52: 213-221, (2014).

[19] Merris, R., "A survey of graph Laplacians", Lin. Multilin. Algebra, 39:19-31, (1995).

[20] Gutman, I., Zhou, B., "Laplacian energy of a graph", Lin. Algebra Appl., 414: 29-37, (2006).

[21] Andriantiana, E.O.D., "Laplacian energy", In: Gutman, I., Li, X. ed., Energies of Graphs -Theory and Applications, Univ. Kragujevac, Kragujevac, 49-80, (2016).

[22] Pirzada, S., Ganie, H.A., "On the Laplacian eigenvalues of a graph and Laplacian energy" Lin. Algebra Appl., 486: 454-468, (2015).

[23] Ganie, H.A., Pirzada, S., Baskoro, E. T., “On energy, Laplacian energy and p-fold graphs”, El. J. Graph Theory Appl., 3: 94-107, (2015).

[24] Liu, J., Liu, B., Radenković, S., Gutman, I., "Minimal LEL-equienergetic graphs", MATCH Commun. Math. Comput. Chem., 61: 471-478, (2009).

[25] Pirzada S., Ganie, H.A., "On the construction of L-equienergetic graphs", AKCE International J. Graphs Combin., 12: 141-154, (2015).

[26] Stevanović, D., "Large sets of noncospectral graphs with equal Laplacian energy", MATCH Commun. Math. Comput. Chem., 61: 463-470, (2009).

[27] Horn, R.A., Johnson, C.R., Matrix Analysis, Cambridge Univ. Press, Cambridge, (1985). 
[28] Liu, J., Liu, B., "Generalization for Laplacian energy", Appl. Math. J. Chinese Univ., 24: 443-450, (2009).

[29] Altindag, I., "Some statistical results for Randić energy of graphs", MATCH Commun. Math. Comput. Chem., 79: 331-339, (2018).

[30] Zumstein, P., "Comparison of spectral methods through the adjacency matrix and the Laplacian of a graph", Diploma Thesis, ETH Zurich, (2005).

[31] Bozkurt, S.B., Gungor, A.D., Gutman, I., Cevik, A.S., "Randić matrix and Randić energy", MATCH Commun. Math. Comput. Chem., 64: 239-250, (2010).

[32] Zhou, B., Gutman, I., "On Laplacian energy of graphs", MATCH Commun. Math. Comput. Chem., 57: 211-220, (2007).

[33] Ganie, H.A., Chat, B. A., Pirzada, S., "Signless Laplacian energy of a graph and energy of a line graph." Lin. Algebra Appl., 544: 306-324, (2018).

[34] Ganie, H.A., Pirzada, S., "On the bounds for signless Laplacian energy of a graph" Discrete Appl. Math., 228: 3-13, (2017). 